J Thromb Haemost. 2016 December ; 14(12): 2353-2355. doi:10.1111/jth.13506.

\title{
Microangiopathic hemolytic anemia due to ADAMTS-13 loss in idiopathic systemic capillary leak syndrome
}

\author{
D. C. MOREIRA ${ }^{\star}$, C. J. NG ${ }^{\star}$, R. QUINONES ${ }^{\star}$, X. LIANG ${ }^{\dagger}$, D. W. CHUNG ${ }^{\ddagger}$, and J. DI PAOLA ${ }^{\star}, \S$ \\ * Department of Pediatrics, University of Colorado Anschutz Medical Campus (AMC) \\ † Department of Pathology, University of Colorado, AMC, Aurora, CO \\ ‡ Bloodworks Research Institute -Puget Sound, Seattle, WA \\ $\S$ Human Medical Genetics and Genomics Program, University of Colorado, Aurora, CO, USA
}

\section{Summary.}

Idiopathic systemic capillary leak syndrome (SCLS) is a rare process characterized by acute and recurrent episodes of vascular leakage with severe hypotension, hypoalbuminemia, hemoconcentration and edema. Anemia and thrombocytopenia are not part of this syndrome, but here we present the case of a pediatric patient with a clinical presentation consistent with SCLS who subsequently developed microangiopathic hemolytic anemia at a time when she had significant fluid loss and anasarca. Based on serial ADAMTS-13 levels, we propose that the anemia in this patient developed as a result of ADAMTS-13 loss in the third-space fluid, a novel mechanism for acquired microangiopathic hemolytic anemia.

\section{Keywords}

ADAMTS-13 protein, human; hemolysis; microangiopathic hemolytic anemia; systemic capillary leak syndrome; thrombocytopenia

\section{Introduction}

Idiopathic systemic capillary leak syndrome (SCLS) is characterized by acute and recurrent episodes of vascular leakage with severe hypotension, hypoalbuminemia, hemoconcentration and edema [1]. It is estimated that as much as $70 \%$ of the intravascular volume can leak out during acute episodes [2]. SCLS is associated with significant mortality and has a 5-year overall survival of approximately $70 \%$ [2]. Since the disease was first described by Clarkson in 1960, less than 300 cases have been reported, most of them being described in

Correspondence: Jorge Di Paola, University of Colorado AMC, Mail Stop 8302, Building RC-1 North, 12800 E. 19th Ave, Aurora, CO 80045, USA. Tel.: +1 303724 4000; fax: +1 303724 4015. jorge.dipaola@ucdenver.edu.

Disclosure of Conflict of Interests

C. J. Ng reports grants and personal fees from CSL Behring and personal fees from Baxalta, outside the submitted work. The other authors state that they have no conflict of interest.

Addendum

D. C. Moreira, R. Quinones, X. Liang, and J. Di. Paola provided clinical care for the patient. D. W. Chang performed laboratory measurements. D. C. Moreira, C. J. Ng, and J. Di Paola wrote the manuscript. All authors reviewed the manuscript. 
adults [3]. The specific underlying mechanism of this condition remains to be elucidated but it is thought that capillary hyper-permeability and subsequent plasma extravasation trigger the clinical presentation [1]. The largest case series in pediatrics found an association with a flulike prodrome and elevated levels of inflammatory cytokines [1,4]. There are no unifying treatment modalities for SCLS, but adult case series have established possible therapeutic interventions, including intravenous immunoglobulin (IVIG) [5]. Hemoconcentration may be also seen secondary to intravascular loss, but changes in total leukocyte or platelet counts are typically not part of SCLS [2]. Here we present the case of a pediatric patient with a clinical presentation consistent with SCLS who then developed microangiopathic hemolytic anemia. We are proposing that this anemia is the result of ADAMTS-13 (a disintegrin and metalloproteinase with a thrombospondin type 1 motif, member 13) loss in the third-space fluid, a novel mechanism for acquired microangiopathic hemolytic anemia.

A previously healthy 5-year-old girl presented with 1 day of severe, intermittent periumbilical abdominal pain, non-bloody and non-bilious emesis but no diarrhea or fever. Physical examination showed abdominal distention with mild lower abdominal tenderness and edema of her hands and feet. Her initial laboratory evaluation was significant for leukocytosis $\left(31.4 \times 10^{3}\right.$ cells $\left.\mu \mathrm{L}^{-1}\right)$ with neutrophilic predominance ( $\left.89 \%\right)$, polycythemia (hemoglobin $\left.18.5 \mathrm{~g} \mathrm{dL}^{-1}\right)$, normal platelet count $\left(433 \times\right.$ cell $\left.\mu \mathrm{L}^{-1}\right)$, normal electrolytes other than mild hyponatremia $\left(133 \mathrm{mmol} \mathrm{L}^{-1}\right)$, hypoalbuminemia $\left(1.9 \mathrm{~g} \mathrm{dL}^{-1}\right)$, normal creatinine $\left(0.6 \mathrm{mg} \mathrm{dL}^{-1}\right)$ and a normal liver function panel (AST $65 \mathrm{U} \mathrm{L}^{-1}$, ALT $23 \mathrm{U} \mathrm{L}^{-1}$, total bilirubin $0.6 \mathrm{mg} \mathrm{dL}^{-1}$ ). An abdominal computed tomography (CT) with intravenous and rectal contrast showed a moderate amount of ascites, but no other intra-abdominal pathology. She was initially admitted to the general pediatrics floor but was transferred to the pediatric intensive care unit because of persistent tachycardia despite fluid resuscitation. Abdominal paracentesis was performed to further evaluate the ascites, which established the presence of acute inflammatory cells of neutrophilic predominance, but no microorganisms. After the procedure, she developed anasarca and bilateral pleural effusions with associated respiratory failure that required intubation and the placement of bilateral chest tubes. She had persistent hypoalbuminemia and was also found to have marked hypogammaglobulinemia, with an initial value of $68 \mathrm{mg} \mathrm{dL}^{-1}$ that was minimally responsive to replacement with IVIG.

Approximately 1 week into her hospital stay and during the time of significant fluid loss (up to $200 \mathrm{~mL} \mathrm{~kg}^{-1} \mathrm{day}^{-1}$ ) from her chest and peritoneal tubes, her hemoglobin level dropped abruptly (trough, $5.9 \mathrm{~g} \mathrm{dL}^{-1}$ ) (Fig. 1B) and review of her peripheral blood smear demonstrated schistocytes, helmet cells and microspherocytes (Fig. 1A). At this time she also had a marked decrease in the platelet count (trough, $18 \times 10^{3}$ cells $\mu \mathrm{L}^{-1}$ ). Because of the presence of a microangiopathic hemolytic anemia, an ADAMTS-13 activity assay was obtained, which showed $9 \%$ activity with no identifiable neutralizing antibody. To replete ADAMTS-13, she was given two serial fresh frozen plasma (FFP) infusions at $10 \mathrm{~mL} \mathrm{~kg}^{-1}$ over two consecutive days with marked clinical improvement and an associated increase in ADAMTS-13 levels (Fig. 1C). She had a robust response to the FFP infusions, probably as a result of enzyme replacement and her overall clinical improvement and decrease in capillary leakage. In addition, her hemoglobin and platelet count improved markedly and the amount 
of schistocytes in the peripheral blood smear also decreased. During this time she also received packed red blood cells and platelet transfusions.

Over the course of her admission, her immunoglobulin levels were also significantly low, probably due to protein loss. She was tested for known mutations that have been associated with atypical HUS in the following genes, CFH, CFI, MCP, CFB, CFHR5, C3, THBD, $D G K E, P L G$ and $A D A M T S-13$, but no mutations were found. The patient was on broad spectrum antibiotics and antifungal coverage during her stay. Her stool was negative for shiga-toxin producing organisms and viral studies for cytomegalovirus, Epstein Barr virus, adenovirus, herpes simplex virus, hantavirus, tularemia, Q fever and brucellosis were completed and all returned negative with the exception of rhinovirus/enterovirus positivity from a nasal swab. She was afebrile with negative cultures throughout the entire hospitalization. She underwent extensive evaluation to establish the origin of her complex clinical presentation, including esophagogastroduodenoscopy, colonoscopy, bone marrow aspirate and positron emission tomography scan, all of which were unremarkable. Because of her persistent hypoalbuminemia and anasarca with no other identifiable cause, she was diagnosed as having SCLS. After inpatient rehabilitation, the patient was discharged home in a stable clinical condition and with normal blood counts.

In the case presented here, we report a potential novel mechanism of microangiopathic hemolytic anemia due to ADAMTS-13 loss. Acquired ADAMTS-13 functional deficiency is typically secondary to antibodies that inhibit its enzymatic activity. The congenital deficiency of ADAMTS-13 (Upshaw-Schulman syndrome) due to mutations in ADAMTS-13, although rare, has a variable presentation and was also considered in the differential diagnosis [6]. Reduced ADAMTS-13 levels have been reported in some adults and children with sepsis and sepsis-like syndromes and have been associated with increased mortality, suggesting that reduced ADAMTS-13 levels may play a role in poor outcomes [7,8]. However, although ADAMTS-13 activity has been proven to be low in some patients with sepsis, the levels reported are usually significantly higher than the ones observed in this patient [9].

In the patient presented here, anti-ADAMTS-13 antibodies were not detected and the level of ADAMTS-13 was normal once the episode of SLCS resolved. Severe ADAMTS-13 deficiency is typically defined as an activity of the protease below $10 \%$ of normal [10]. Although in the initial presentation the patient showed no signs of hemolysis and had a normal platelet count, during the worsening of her clinical status, which was associated with high fluid loss, she developed microangiopathic hemolytic anemia. By sequential measurements, we hypothesized that the decrease of ADAMTS-13 was likely to be secondary to non-specific protein loss in the transudate because the timing of decreased ADAMTS-13 activity correlated with decreased levels of IgG, which has been known to be lost in transudate fluid. Unfortunately, we did not have access to the transudate fluid to test for ADAMTS-13 levels. However, further supporting this hypothesis is the similar molecular weight of $\operatorname{IgG}(150 \mathrm{kDa})$ and ADAMTS-13 $(154 \mathrm{kDa})$, suggesting that they may be lost at a similar rate and perhaps through a similar mechanism $[11,12]$. It has been proposed that molecules with a molecular weight of less than $200 \mathrm{kDa}$ leak out in SCLS [2]. Further clinical data supporting this hypothesis are the improvement in levels of ADAMTS-13 with 
the infusion of FFP and the improvement in IgG level with infusions of IVIG. We cannot rule out a decrease in ADAMTS-13 production in the liver; however, the liver's synthetic function in this patient did not appear to be affected.

In summary, we propose a novel mechanism of ADAMTS-13 loss that can lead to microangiopathic hemolytic anemia in patients with significant loss of proteins into the extravascular space in clinical conditions such as SCLS.

\section{References}

\section{References}

1. Hsu P, Xie Z, Frith K, Wong M, Kakakios A, Stone KD, Druey KM. Idiopathic systemic capillary leak syndrome in children. Pediatrics 2015; 135: 730-5.

2. Dhir V, Arya V, Malav IC, Suryanarayanan BS, Gupta R, Dey AB. Idiopathic systemic capillary leak syndrome (SCLS): case report and systematic review of cases reported in the last 16 years. Intern Med 2007; 46: 899-904. [PubMed: 17575386]

3. Clarkson B, Thompson D, Horwith M, Luckey EH. Cyclical edema and shock due to increased capillary permeability. Am J Med 1960; 29: 193-216. [PubMed: 13693909]

4. Xie Z, Chan E, Yin Y, Ghosh CC, Wisch L, Nelson C, Young M, Parikh SM, Druey KM. Inflammatory markers of the systemic capillary leak syndrome (Clarkson Disease). J Clin Cell Immunol 2014; 5: 1000213.

5. Lambert M, Launay D, Hachulla E, Morell-Dubois S, Soland V, Queyrel V, Fourrier F, Hatron PY. High-dose intravenous immunoglobulins dramatically reverse systemic capillary leak syndrome. Crit Care Med 2008; 36: 2184-7. [PubMed: 18552679]

6. Pérez-Rodríguez A, Lourés E, Rodríguez-Trillo Á, Costa-Pinto J, García-Rivero A, Batlle-López A, Batlle J, López-Fernández MF. Inherited ADAMTS-13 deficiency (Upshaw-Schulman syndrome): a short review. Thromb Res 2014; 134: 1171-5. [PubMed: 25242241]

7. Bongers TN, Emonts M, de Maat MP, de Groot R, Lisman T, Hazelzet JA, Leebeek FW. Reduced ADAMTS-13 in children with severe meningococcal sepsis is associated with severity and outcome. Thromb Haemost 2010; 103: 1181-7. [PubMed: 20390223]

8. Fukushima H, Nishio K, Asai H, Watanabe T, Seki T, Matsui H, Sugimoto M, Matsumoto M, Fujimura Y, Okuchi K. Ratio of von Willebrand factor propeptide to ADAMTS-13 is associated with severity of sepsis. Shock 2013; 39: 409-14. [PubMed: 23481506]

9. Bianchi V, Robles R, Alberio L, Furlan M, Lämmle B. Von Willebrand factor-cleaving protease (ADAMTS-13) in thrombocytopenic disorders: a severely deficient activity is specific for thrombotic thrombocytopenic purpura. Blood 2002; 100: 710-3. [PubMed: 12091372]

10. Lotta LA, Wu HM, Musallam KM, Peyvandi F. The emerging concept of residual ADAMTS-13 activity in ADAMTS-13-deficient thrombotic thrombocytopenic purpura. Blood 2013; 27: 71-6.

11. Janeway CA Jr, Travers P, Walport M, Shlomchik MJ. Immunobiology: The Immune System in Health and Disease, 5th edn New York: Garland Science, 2001.

12. Furlan M Proteolytic cleavage of von Willebrand factor by ADAMTS-13 prevents uninvited clumping of blood platelets. J Thromb Haemost 2004; 2: 1505-9. [PubMed: 15333021] 


\section{Essentials}

- $\quad$ Idiopathic systemic capillary leak syndrome (SCLS) is characterized by episodes of vascular leakage.

- We present the case of a patient with SCLS who developed microangiopathic hemolytic anemia (MAHA).

- We propose that this anemia is the result of ADAMTS-13 loss in the thirdspace fluid.

- $\quad$ This suggests that MAHA can occur in patients with significant extravasation of proteins. 
A
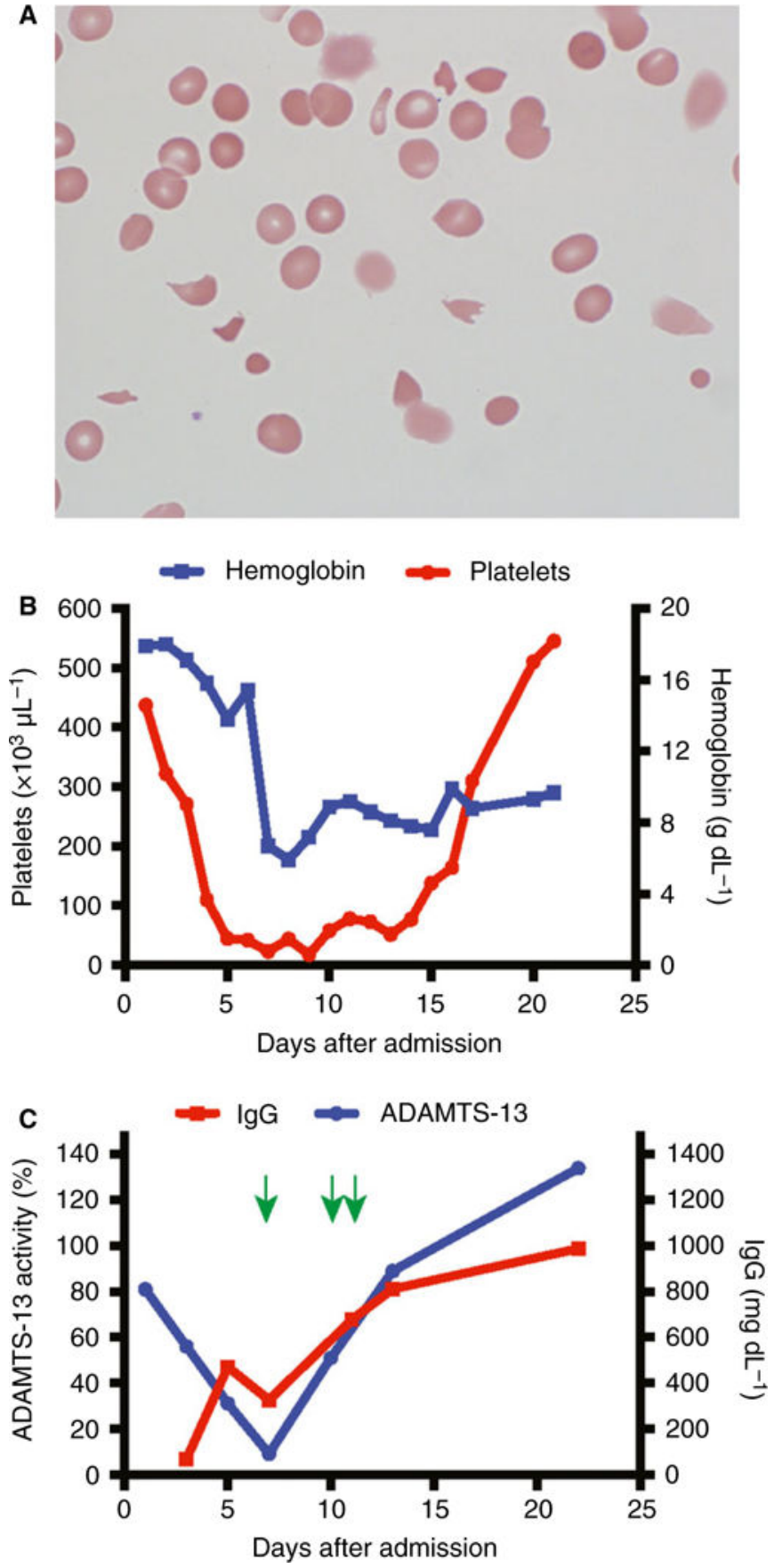

Fig. 1.

ADAMTS-13 levels mimic IgG levels and coincide with the nadir of hemoglobin and platelets. (A) A representative image of the patient's peripheral blood smear stained with Wright-Giemsa (100X) at the time of 9\% ADAMTS-13 level. (B) Values of hemoglobin and platelets over the hospital admission period. (C) The serial values of ADAMTS-13 and IgG over the time of hospital admission. Green arrows illustrate time of fresh frozen plasma (FFP) administration. 
[Color figure can be viewed at wileyonlinelibrary.com]. 